Article

\title{
Polyhexamethylene Biguanide and Nadifloxacin Self-Assembled Nanoparticles: Antimicrobial Effects against Intracellular Methicillin-Resistant Staphylococcus aureus
}

\author{
Nor Fadhilah Kamaruzzaman ${ }^{1,2, *(1)}$, Maria de Fatima Pina ${ }^{3}$, Alexandru Chivu ${ }^{4}$ and Liam Good ${ }^{1}$ \\ 1 Department of Pathobiology and Population Science, Royal Veterinary College, University of London, \\ Royal College Street, London NW1 0TU, UK; lgood@rvc.ac.uk \\ 2 Present address: Faculty of Veterinary Medicine, Universiti Malaysia Kelantan, Locked Bag 36, \\ Pengkalan Chepa, 16100 Kota Bharu, Kelantan, Malaysia \\ 3 University College London School of Pharmacy, 29-39 Brunswick Square, Bloomsbury, London WC1N 1AX, \\ UK; mfatimagpina@gmail.com \\ 4 UCL Centre for Nanotechnology and Regenerative Medicine, Division of Surgery \& Interventional Science, \\ University College London, London NW3 2PF, UK; a.chivu.14@ucl.ac.uk \\ * Correspondence: norfadhilah@umk.edu.my; Tel.: +609-7717334
}

Received: 24 March 2018; Accepted: 9 May 2018; Published: 12 May 2018

check for updates

\begin{abstract}
The treatment of skin and soft tissue infections caused by methicillin-resistant Staphylococcus aureus (MRSA) remains a challenge, partly due to localization of the bacteria inside the host's cells, where antimicrobial penetration and efficacy is limited. We formulated the cationic polymer polyhexamethylene biguanide (PHMB) with the topical antibiotic nadifloxacin and tested the activities against intracellular MRSA in infected keratinocytes. The PHMB/nadifloxacin nanoparticles displayed a size of $291.3 \pm 89.6 \mathrm{~nm}$, polydispersity index of $0.35 \pm 0.04$, zeta potential of $+20.2 \pm 4.8 \mathrm{mV}$, and drug encapsulation efficiency of $58.25 \pm 3.4 \%$. The nanoparticles killed intracellular MRSA, and relative to free polymer or drugs used separately or together, the nanoparticles displayed reduced toxicity and improved host cell recovery. Together, these findings show that PHMB/nadifloxacin nanoparticles are effective against intracellular bacteria and could be further developed for the treatment of skin and soft tissue infections.
\end{abstract}

Keywords: skin and soft tissue infections; polyhexamethylene biguanide; nadifloxacin; nanoparticles; intracellular MRSA

\section{Introduction}

Skin and soft-tissue infections (SSTIs) affect a wide range of patients including elderly and hospitalized individuals with immunocompromised conditions, and individuals with skin injuries who have frequent skin to skin contact, such as athletes [1,2]. Clinical manifestation of SSTIs can range from mild to life-threatening infections, with more than $70 \%$ of cases requiring hospitalization [3].

Staphylococcus aureus (S. aureus) is the leading pathogen causing SSTIs worldwide [4-6]. Staphylococcus aureus is a Gram-positive pathogen that colonizes between 15 to $40 \%$ of healthy individuals in their nose, skin, and mucous membranes [7]. Infections by S. aureus were once easy to treat by the administration of beta-lactam antibiotics. However, the rise of methicillin-resistant S. aureus (MRSA)-associated infections worldwide has led to resistance against available antibiotic therapy, increasing morbidity and mortality in hospitalized patients $[8,9]$. Additionally, S. aureus is known to invade and survive in the host's cell, and in this state, they are further protected from the 
immune surveillance and antimicrobial therapy. These protection mechanisms can lead to persistent infections, which may increase transmission and mortality [10]. Many antimicrobials do not effectively treat intracellular infections due to (1) poor penetration and retention of the host cells; (2) reduced potency in intracellular acidic environments and localization in the different cell compartments where bacteria reside; and (3) reduced potency towards slow-growing intracellular bacteria [11-16]. These factors are believed to contribute to therapeutic failure.

The development of a new antimicrobial is a lengthy and expensive process. An alternative approach is to improve the overall potency of available antimicrobials through formulation. Advances in nanotechnology offer new possibilities to improve potency and reduce toxicity. Nanoparticles can be prepared using synthetic or natural substances and their features include large surface areas and functional chemical groups that can be utilized in combination with active pharmaceutical ingredients (APIs) [17]. Physical encapsulation, adsorption or chemical conjugation of drugs into nanoparticles can significantly improve the therapeutic index [18]. For example, Miramoth et al. [19] demonstrated that squalenoylated penicillin nanoparticles are more effective against intracellular $S$. aureus in macrophages in comparison to free penicillin. Clemens et al. [20] showed that rifampicin loaded into mesoporous silica-polyethyleneimine nanoparticles are more potent towards intracellular Mycobacteria tuberculosis, in comparison to the free drug. Such examples demonstrate that combinations of antimicrobials in nanoparticulate formulations can potentially improve antimicrobial potency. Additional advantages of the application of nanoparticles for drug loading and delivery have been described in References [21-27].

We recently reported that polyhexamethylene biguanide (PHMB)—a cationic antimicrobial polymer (see Figure 1a) — can bind to nucleic acids, forming nanoparticles in vitro [28]. Additionally, we demonstrated that PHMB can form nanoparticles and improve delivery of CpG oligonucleotide $(\mathrm{ODN})$, an immunomodulatory, into macrophages [29,30]. We also demonstrated that the polymer can enter a range of mammalian cells and kill intracellular bacteria and parasites [31]. Therefore, PHMB characteristics provide an exciting platform for formulation with antimicrobials, potentially improving antimicrobial cell delivery and controlled release, as well as providing direct polymer-mediated antimicrobial effects. We also showed that nadifloxacin (Figure 1b) -a topical antimicrobial that is currently administered for the treatment of skin infections-has antimicrobial activities against intracellular S. aureus [31]. With this knowledge, we aimed to further exploit PHMB by examining its possible interaction with nadifloxacin to form nanoparticles, and to further determine the antimicrobial activities of the nanoparticles against intracellular $S$. aureus in infected keratinocytes and to characterize the toxicity profile against the host cells.

a)

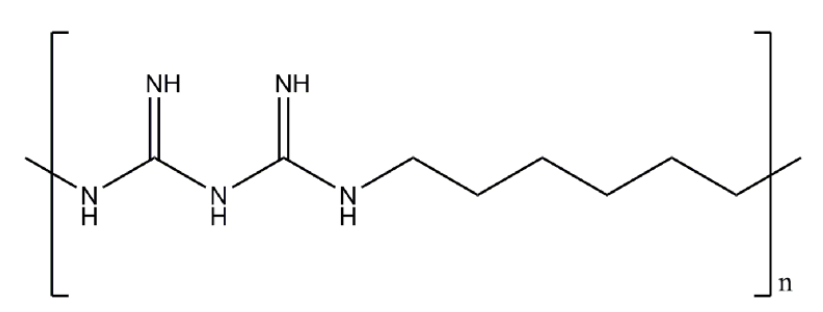

b)<smiles>CC1CCc2c(N3CCC(O)CC3)c(F)cc3c(=O)c(C(=O)O)cn1c23</smiles>

Figure 1. Structure of (a) that polyhexamethylene biguanide (PHMB) and (b) nadifloxacin. 
Polyhexamethylene biguanide is a cationic polymer of repeating hexamethylene biguanide groups, with $n$ average $=10-12$ ( $n$ is the number of structural unit repeats) and a molecular weight of $(M W)$ $3025 \mathrm{~g} / \mathrm{mol}$. Nadifloxacin is a topical fluoroquinolone with $M W 360 \mathrm{~g} / \mathrm{mol}$.

\section{Materials and Methods}

\subsection{Preparation and Optimization of PHMB/Nadifloxacin Nanoparticles}

Polyhexamethylene biguanide was obtained from Tecrea Ltd., London, UK and nadifloxacin was obtained from Santa Cruz Biotechnology, UK. All antimicrobials were prepared in stock solution at $10 \mathrm{mg} / \mathrm{mL}$. PHMB was dissolved in sterile distilled water, and nadifloxacin was dissolved in $0.1 \mathrm{M}$ sodium hydroxide solution.

The PHMB/nadifloxacin nanoparticles were prepared by the self-assembly method [28]. In brief, $0.5 \mathrm{~mL}$ of PHMB $(80 \mathrm{mg} / \mathrm{L}$ in water) and $0.5 \mathrm{~mL}$ of nadifloxacin $(160 \mathrm{mg} / \mathrm{L}$ in $0.1 \mathrm{M} \mathrm{NaOH})$ were mixed in a $1.5 \mathrm{~mL}$ microcentrifuge tube followed by incubation in an incubator shaker at $200 \mathrm{rpm}$ for $15 \mathrm{~min}$.

\subsection{Physical Characterization of PHMB/Nadifloxacin Nanoparticles}

\subsubsection{Size and Zeta Potential Measurements}

Intensity mean hydrodynamic size and zeta potential of PHMB and PHMB/nadifloxacin nanoparticles were measured on a Malvern Zetasizer-NanoZS (Malvern Instruments, Malvern, UK) with a He-Ne laser (wavelength of $632.8 \mathrm{~nm}$ ). The measurements were carried out at a scattering angle of 173 at $25^{\circ} \mathrm{C}$.

\subsubsection{Transmission Electron Microscopy (TEM)}

Transmission Electron Microscopy images were collected using a JEOL-1010 (JEOL Ltd., Tokyo, Japan), equipped with a side-mounted Gatan Orius CCD digital camera. A drop of the nanoparticles suspension was placed on a 400-mesh formvar-coated carbon grid (Agar Scientific, Stansted, UK), followed by staining with $2 \%$ phosphotungstate acid.

\subsubsection{Encapsulation Efficiency}

The encapsulation efficiency was determined as described in Reference [32]. Free nadifloxacin concentrations were measured in the recovered medium after particle centrifugation using a Sigma 3-16 centrifuge (Sigma, Osteorode am Harz, Germany) at $5000 \mathrm{rpm}$ for $15 \mathrm{~min}$. Amicon Ultra-4 centrifugal filter units with ultracel-3 membrane MW cut-off of $50 \mathrm{kDa}$ were used. The nadifloxacin concentration collected after centrifugation (non-encapsulated nadifloxacin) was measured using UV spectroscopy at $290 \mathrm{~nm}$. The nadifloxacin encapsulation efficiency (EE) (\%) was given by the difference between the total amount of nadifloxacin added for the nanoparticles preparation, and the nadifloxacin collected in solution after centrifugation to the total amount of nadifloxacin added.

\subsection{Bacterial Strains and Growth Conditions}

S. aureus strain EMRSA-15 was obtained from Dr. Sean Nair, University College, London. Bacteria were grown in Mueller Hinton Broth (MHB) (Sigma-Aldrich, Dorset, UK) followed by incubation at $250 \mathrm{rpm}$ (for liquid cultures), at $37^{\circ} \mathrm{C}$ for $18 \mathrm{~h}$. 


\subsection{Eukaryotic Cell Lines and Growth Conditions}

HaCaT cells were obtained from Dr. Amir Sharili, Queen Mary University, London and maintained in DMEM with 10\% fetal bovine serum (FBS) (Sigma-Aldrich, Dorset, UK), supplemented with $5 \%$ penicillin-streptomycin (Sigma-Aldrich, Dorset, UK). Cells were maintained at $37{ }^{\circ} \mathrm{C}$ in $5 \%$ carbon dioxide.

\subsection{Intracellular Infection of Keratinocytes by MRSA}

Briefly, keratinocytes were seeded at $1.2 \times 10^{5}$ cells/well in a 12-well plate and cultured overnight in DMEM with 10\% FBS, without antibiotic. In parallel, MRSA strain EMRSA-15 was cultured overnight in $\mathrm{MHB}$ at $37^{\circ} \mathrm{C}$ in an incubator shaker. One $\mathrm{mL}$ of overnight bacterial culture was centrifuged at $8000 \mathrm{rpm}$ for three minutes and the pellet was resuspended in phosphate buffered saline (PBS) (Sigma-Aldrich, Dorset, UK). These steps were repeated three times to remove the bacterial toxin residues. Bacteria were diluted to a final concentration of approximately $10^{7} \mathrm{CFU} / \mathrm{mL}$ in DMEM with $10 \%$ FBS, without antibiotic. Aliquots of bacteria $\left(1 \mathrm{~mL}, 10^{7} \mathrm{CFU} / \mathrm{mL}\right)$ were added to keratinocyte cultures after the original medium was removed. Bacteria were co-incubated with keratinocytes for three hours. Two hundred $\mathrm{mg} / \mathrm{L}$ of gentamicin diluted in the medium was added and incubated for three hours. Medium containing bacteria and gentamicin were removed and cells were rinsed with PBS. A rinse step was performed because this medium contained very high concentrations of gentamicin, and therefore did not result in colony-forming units of bacteria when plated directly. Therefore, aliquots of PBS from the rinsing process were plated on nutrient agar to determine the remaining number of extracellular bacteria. Next, one $\mathrm{mL}$ of $0.5 \%$ Triton X-100 prepared in PBS was added to each well to lyse cells. Lysed cells were serially diluted in PBS and plated on nutrient agar (Sigma-Aldrich, Dorset, UK) for enumeration of intracellular bacteria. Uninfected cells were also subjected to the lysis procedure to confirm sterility.

\subsection{Antimicrobial Activities of PHMB/Nadifloxacin Nanoparticles against Intracellular MRSA}

Keratinocytes were infected with MRSA using gentamicin protection assay as described in Section 2.5. Following gentamicin exposure to kill extracellular bacteria, infected cells were treated with PHMB and nadifloxacin alone, a combination of PHMB and nadifloxacin added individually to the wells, and PHMB/nadifloxacin pre-formulated as nanoparticles for three hours. Cells were then lysed with $0.5 \%$ Triton X-100. Lysed cells were serially diluted in PBS and plated on nutrient agar (Sigma-Aldrich, Dorset, UK) for enumeration of intracellular bacteria. Uninfected cells were also subjected to the lysis procedure to confirm sterility.

\subsection{Assessment Re-Growth of MRSA}

Keratinocytes were infected with MRSA, treated with gentamicin, followed by treatment with different antimicrobial formulations: PHMB and nadifloxacin alone or in combination as described above, for $24 \mathrm{~h}$ for over $72 \mathrm{~h}$ of the experiment. For every $24 \mathrm{~h}$, old medium containing antimicrobials was removed and plated for colony counting and replaced with fresh medium containing antimicrobials.

\subsection{Assessment Recovery of Infected Keratinocytes}

After 72 h, cells were imaged by a DM4000B (Leica Biosystem, Wetzlar, Germany) upright microscope with the $20 \times$ objective lens. The morphology of keratinocytes was observed to evaluate recovery following infection and treatment. Also, to estimate cell viability, cells were trypsinized and counted using a hemacytometer. 


\subsection{Assessment of Nanoparticle Toxicity towards Keratinocytes}

\subsubsection{LDH Cytotoxicity Assay}

Keratinocytes were seeded at $1.2 \times 10^{5}$ cells /well in a 12-well plate and cultured overnight in DMEM with 10\% FBS, without antibiotic. Keratinocytes were exposed to increasing concentrations of PHMB and nadifloxacin alone and in combinations, for $24 \mathrm{~h}$. After $24 \mathrm{~h}, 100 \mu \mathrm{L}$ of medium were taken for measurement of LDH released by the cells using Pierce LDH ${ }^{\mathrm{TM}}$ Cytotoxicity Assay Kit (Thermo-Scientific, Rugby, UK). LDH assay was performed as described by the manufacturer.

\subsubsection{Resazurin Cell Viability Assay}

A resazurin assay was performed following $\mathrm{LDH}$ toxicity assay. The resazurin sodium salt (Sigma-Aldrich, Dorset, UK) was prepared as a stock solution at $440 \mu \mathrm{M}$ in PBS and added to each well at $44 \mu \mathrm{M}$ final concentrations. Plates were incubated for an additional $24 \mathrm{~h}$. Optical density (OD) was then measured using a Tecan Infinite plate reader (Tecan Group Ltd., Mannedorf, Switzerland) at $550 \mathrm{~nm}$ and $630 \mathrm{~nm}$. The OD value change (or \% dye reduction) proportional to the viable cell number was used to plot the graph.

\subsection{Statistical Analysis}

All experiments were performed in triplicate. Statistical analysis was performed using one-way analysis of variance (ANOVA) followed by Tukey tests using the statistical packages Prism 6, Version 6.0 (GraphPad Prism 6.0, San Diego, CA, USA). Data is presented as means \pm standard deviation (SD). Differences were considered to be statistically significant where $p \leq 0.05$. For histogram and graphs, error bars represent standard deviations. ${ }^{*}(p \leq 0.05),{ }^{* *}(p \leq 0.001),{ }^{* * * *}(p \leq 0.0001), \mathrm{ns}$ (not significant).

\section{Results}

\subsection{Physical Characterization of PHMB/Nadifloxacin Nanoparticles}

To prepare the PHMB/nadifloxacin nanoparticles, both compounds were mixed in to a ratio of PHMB to nadifloxacin 1:2 $(w / w)$. The ratio was determined based on the minimum inhibitory concentrations (MIC) of the individual compound against EMRSA-15, where the MIC of PHMB and nadifloxacin were $1 \mathrm{mg} / \mathrm{L}$ and $2 \mathrm{mg} / \mathrm{L}$, respectively [33]. Formulation of PHMB and nadifloxacin produced nanoparticles with Z-average of $291.3 \pm 89.6 \mathrm{~nm}$ (Figure 2a), with a polydispersity index (PDI) of $0.35 \pm 0.04$. The zeta potential of the nanoparticles was found to be positive $(+20.2 \pm 4.83 \mathrm{mV})$, as expected, given that PHMB is a cationic polymer. The nanoparticles at this size have an irregular sphere shape as shown in Figure $2 b$. To measure the encapsulation efficiency, the free nadifloxacin was recovered in the medium following particle centrifugation and subjected to UV spectroscopy at $290 \mathrm{~nm}$. The nadifloxacin encapsulation efficiency in the nanoparticle was determined to be approximately $58 \%$. 
a)

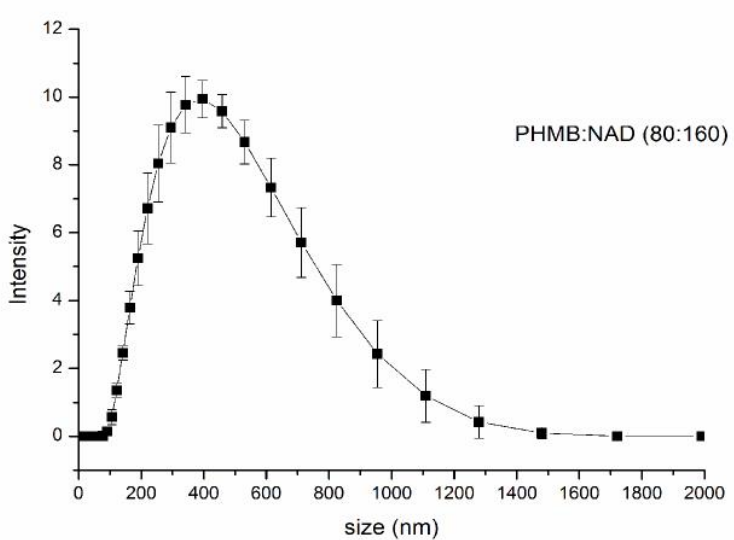

b)

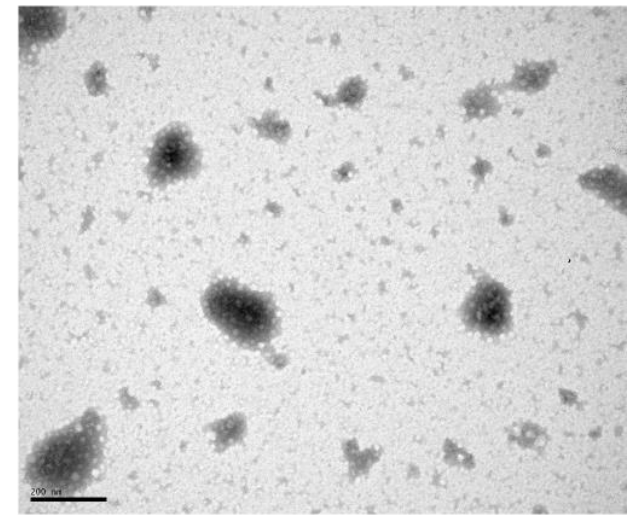

Figure 2. Physical characterization of the PHMB/nadifloxacin nanoparticles. (a) Size distribution profile obtained by dynamic light scattering analysis of $\mathrm{PHMB} /$ nadifloxacin nanoparticles. (b) Transmission electron microscopy (TEM) image of PHMB/nadifloxacin nanoparticles. The scale bar in the TEM image is $200 \mathrm{~nm}$.

\subsection{Intracellular Infections of Keratinocytes by MRSA}

We recently demonstrated that PHMB and nadifloxacin alone could kill intracellular MRSA in keratinocytes [31]. To test whether or not these nanoparticles can retain or improve the antimicrobial effects of the constituents towards intracellular MRSA, the potencies of intracellular antimicrobial effects was determined using a gentamicin protection assay as described [31]. Briefly, keratinocytes were infected with MRSA and exposed to gentamicin to kill extracellular bacteria. The MRSA strain EMRSA-15 showed consistent invasive activities towards keratinocytes, as indicated by its ability to evade gentamicin treatment. Lysis of keratinocytes following gentamicin exposure released approximately $10^{5} \mathrm{CFU} / \mathrm{mL}$ of EMRSA-15, reflecting the bacteria that were inside the keratinocytes (Figure 3).

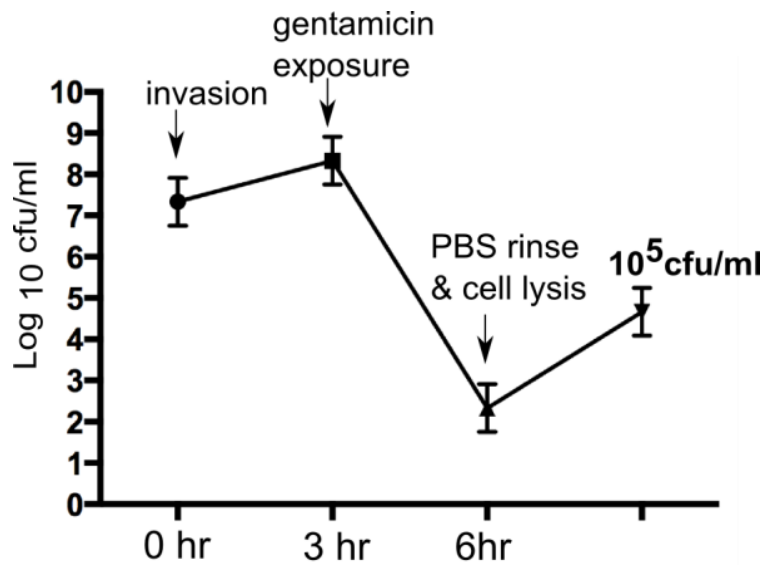

Figure 3. Methicillin-resistant Staphylococcus aureus (MRSA) invasion of keratinocytes.

Colony forming units (CFU) of MRSA following gentamicin exposure. After gentamicin exposure, lysis of keratinocytes released approximately $10^{5} \mathrm{CFU} / \mathrm{mL}$ of EMRSA- 15 . 


\subsection{Antimicrobial Activities of PHMB/Nadifloxacin Nanoparticles against Intracellular MRSA}

To test antimicrobial activities of $\mathrm{PHMB} /$ nadifloxacin nanoparticles against intracellular EMRSA-15 (MRSA), both antimicrobials were tested at two and four times their MIC. Keratinocytes were infected with MRSA, as previously described. Following gentamicin exposure sufficient to kill extracellular bacteria, keratinocytes were treated with the following formulations: PHMB alone (at 2 and $4 \mathrm{mg} / \mathrm{L}$ ); nadifloxacin alone (at 4 and $8 \mathrm{mg} / \mathrm{L}$ ); combination of PHMB (2 mg/L) and nadifloxacin $(4 \mathrm{mg} / \mathrm{L})$, which were added individually into the wells without pre-formulation as nanoparticles; and PHMB/nadifloxacin $(2: 4 \mathrm{mg} / \mathrm{L})$ pre-formulated as nanoparticles. The treatment was performed for three hours. Figure 4 shows the percentages of surviving intracellular MRSA after treatment with the antimicrobial formulations relative to the untreated infected cells. PHMB alone at $2 \mathrm{mg} / \mathrm{L}$ and $4 \mathrm{mg} / \mathrm{L}$ killed $82 \%$ and $99 \%$ of intracellular MRSA, respectively. Nadifloxacin alone at 4 and $8 \mathrm{mg} / \mathrm{L}$ killed $52 \%$ and $70 \%$ of intracellular MRSA, respectively. Combination of PHMB $(2 \mathrm{mg} / \mathrm{L})$ and nadifloxacin $(4 \mathrm{mg} / \mathrm{L}$ ) (added individually into the wells) killed $83 \%$ of intracellular MRSA. Finally, PHMB/nadifloxacin nanoparticles (2:4 mg/L) killed 95\% of intracellular MRSA, and appeared to be more effective than the same concentrations of polymer and drug used alone, or added separately to the same culture (Figure 4); however, it is important to note that the difference was not significant.

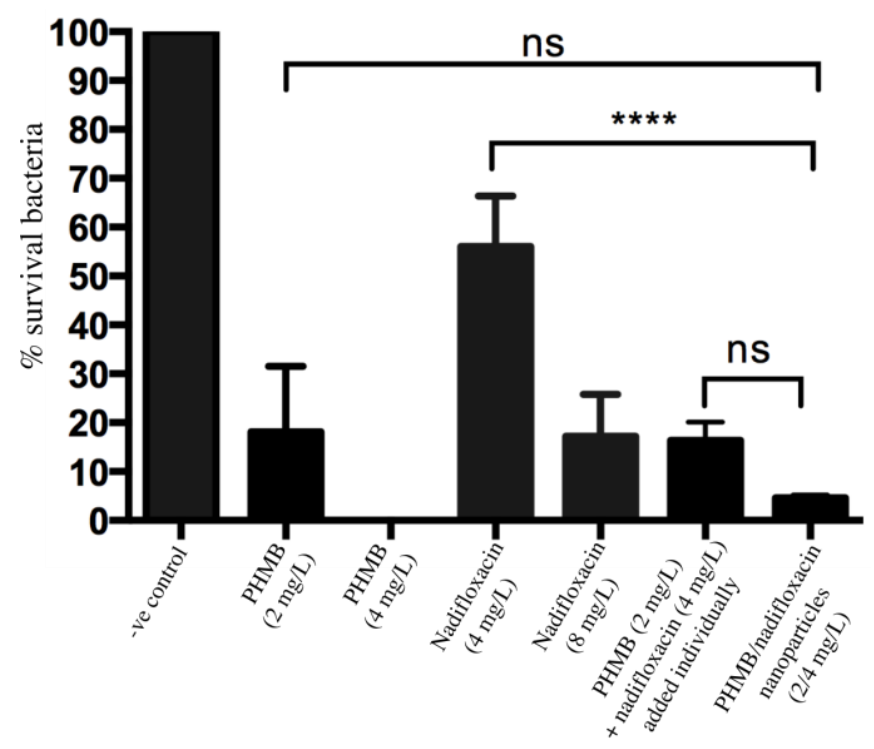

Figure 4. Antimicrobial activities of nanoparticles against intracellular MRSA.

Keratinocytes infected with MRSA were either treated with PHMB or nadifloxacin alone, or in combinations. Combinations of PHMB and nadifloxacin were added individually or pre-formulated as nanoparticles. Untreated cultures were used to establish the CFU values corresponding to $100 \%$ survival. Negative controls were the non-treated infected cells. Error bars represent standard deviations. ${ }^{* * * *}(p \leq 0.0001)$, ns (not significant).

\subsection{Inhibition of MRSA Re-Growth}

To evaluate whether any of the formulations tested in this study were able to prevent re-growth of the bacteria, keratinocytes were infected with MRSA, incubated with gentamicin to kill extracellular bacteria, and treated with antimicrobial formulations as described above for $24 \mathrm{~h}$. At $0,24,48$, and $72 \mathrm{~h}$, the medium containing antimicrobials formulations was sampled, plated for colony counting, and replaced with fresh medium containing antimicrobials. Re-growth of MRSA was observed for infected keratinocytes treated with PHMB alone at $2 \mathrm{mg} / \mathrm{L}$, but not at $4 \mathrm{mg} / \mathrm{L}$. Re-growth 
of MRSA was also observed for treatment with nadifloxacin alone at $4 \mathrm{mg} / \mathrm{L}$ but not at $8 \mathrm{mg} / \mathrm{L}$. Interestingly, combinations of PHMB $(2 \mathrm{mg} / \mathrm{L})$ and nadifloxacin $(4 \mathrm{mg} / \mathrm{L})$, either added individually or pre-formulated as nanoparticles, prevented re-growth of MRSA. Therefore, a combination of PHMB and nadifloxacin at a lower dosage, either added separately as free components or pre-formulated as nanoparticles, were able to kill intracellular MRSA and prevent bacterial re-growth. Figure 5 summarizes the bactericidal activities of antimicrobials formulations following $72 \mathrm{~h}$ of the experiment.

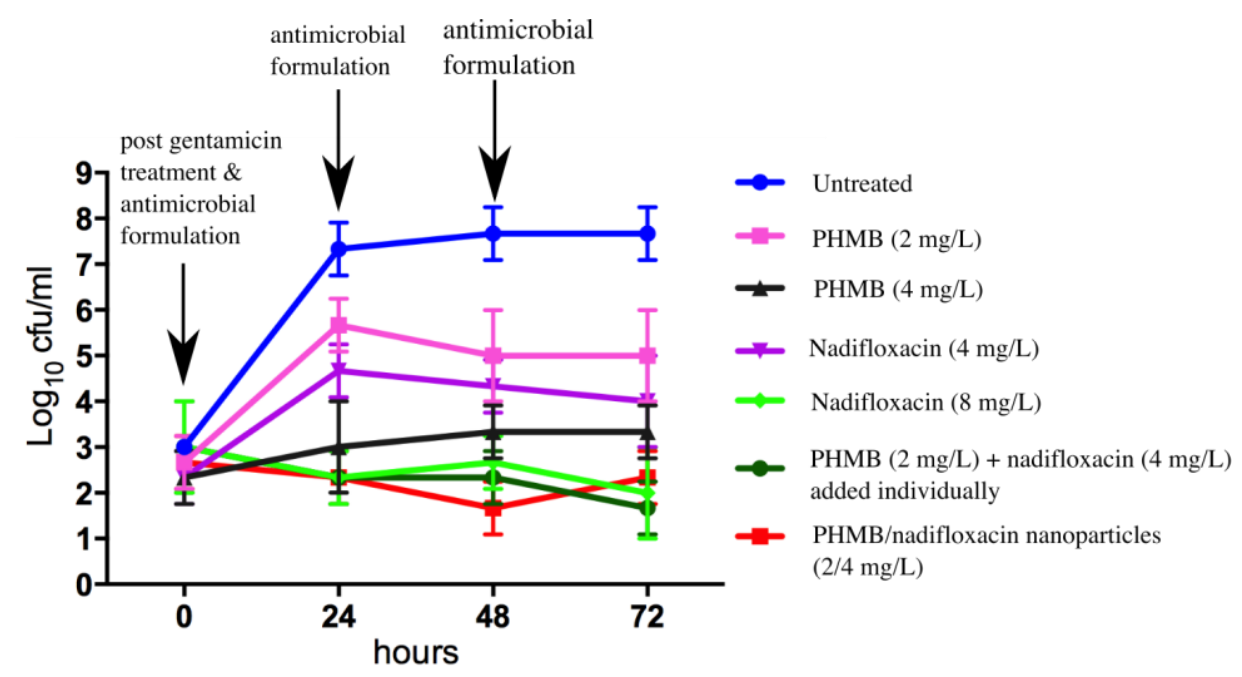

Figure 5. Effects of the different antimicrobials on MRSA re-growth.

Keratinocytes infected with MRSA were either treated with PHMB or nadifloxacin alone or in combination. Combinations of PHMB and nadifloxacin were added individually or pre-formulated as nanoparticles. Untreated cultures were used as a negative control. Every $24 \mathrm{~h}$ for $72 \mathrm{~h}$, the medium containing antimicrobials formulations was sampled, plated for colony counting, and replaced with fresh medium containing antimicrobials.

\subsection{Recovery of Infected Keratinocytes}

Invasion by virulent pathogens such as MRSA can induce pyroptosis (i.e., cell death) of host cells [34]. Therefore, an antimicrobial must not only effectively kill the bacteria, but at the same time it must help host cells recovery, or at least not inhibit this process. During the experiments performed above to investigate the prevention of the re-growth of the bacteria, we monitored the morphology of keratinocytes each day throughout the experiment. After $72 \mathrm{~h}$ of the treatment with different antimicrobial formulations, the recovery of infected keratinocytes was in the following order of rank: PHMB/nadifloxacin nanoparticles $(2: 4 \mathrm{mg} / \mathrm{L})>$ PHMB $(2 \mathrm{mg} / \mathrm{L})$ and nadifloxacin $(4 \mathrm{mg} / \mathrm{L})$ added individually into the wells $>$ PHMB $(4 \mathrm{mg} / \mathrm{L})>$ nadifloxacin $(8 \mathrm{mg} / \mathrm{L})$. It is surprising that cells treated with the nanoparticles showed better recovery in comparison to the cells treated with combinations of PHMB and nadifloxacin added individually into the wells, though both formulations displayed almost equal antimicrobial efficacy. Therefore, PHMB/nadifloxacin nanoparticles not only demonstrate excellent antimicrobial activities against MRSA, but also help improve recovery of infected keratinocytes. Figure 6 shows the morphology and the number of recovered keratinocytes following treatment with different antimicrobial formulations for $72 \mathrm{~h}$. 


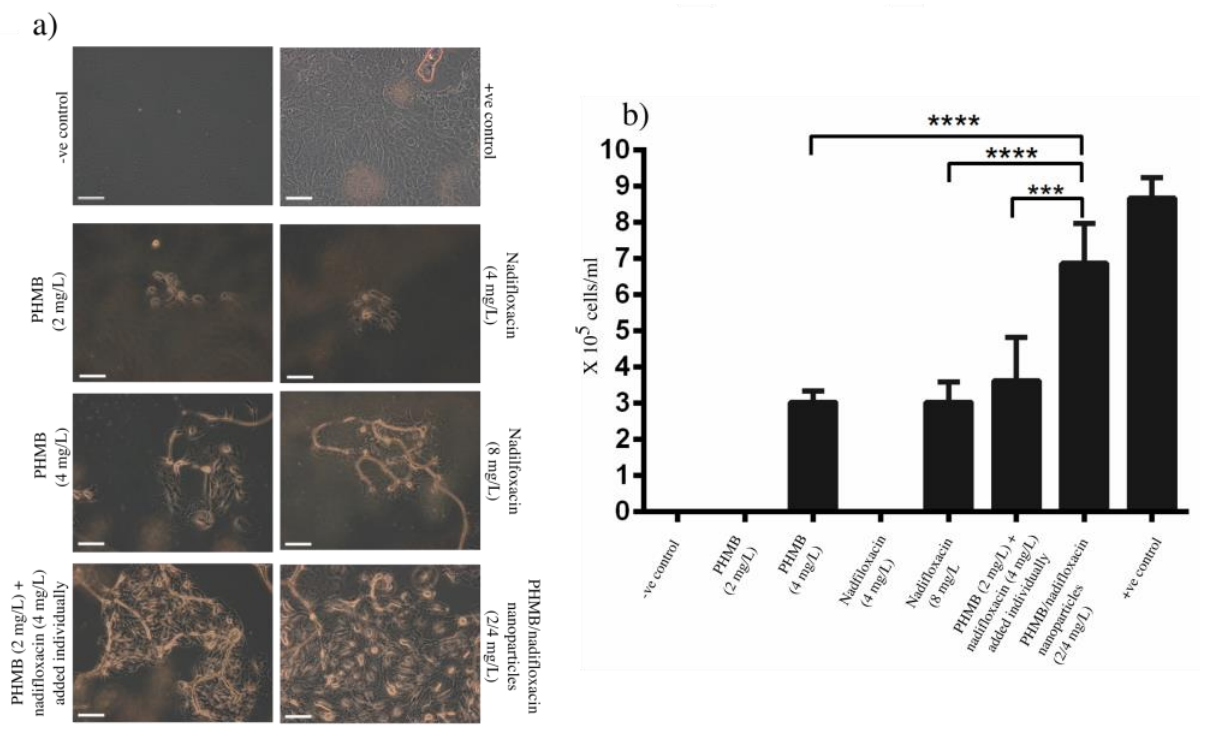

Figure 6. Effects of the antimicrobials on the keratinocyte's recovery. Following antimicrobial treatment for $72 \mathrm{~h}$, infected cells were: (a) visualized by microscopy; and (b) trypsinized and counted using a hemocytometer. The non-infected cells represent the positive control. The scale bar in the image is $100 \mu \mathrm{m}$. Bacteria added to the wells without pre-seeded host cells represent the negative control. Non-treated cells represent positive control. Error bars represent standard deviations. ${ }^{* * *}(p \leq 0.001)$, $* * * *(p \leq 0.0001)$.

\subsection{Toxicity of PHMB/Nadifloxacin Nanoparticles}

To investigate the toxicity of the formulations in relation to the free components, lactate dehydrogenase (LDH) and resazurin assays were performed. Lactate dehydrogenase is a cytoplasmic enzyme, thus, if this enzyme is released into the medium, this would indicate a disruption of the membrane integrity, and therefore a sign of the toxicity effect of the tested compound towards the host cells. In the resazurin assay, metabolically active mitochondria in viable cells reduce resazurin (purple color) into resofurin (pink color). Keratinocytes were exposed to increasing concentrations of PHMB alone (2-64 mg/L), nadifloxacin alone (4-128 mg/L) or in combinations. Combinations of PHMB and nadifloxacin were either added individually or formulated into nanoparticles. For combinations of PHMB and nadifloxacin added separately, the free components were added simultaneously in the well, whereas the nanoparticles were pre-prepared at designated concentrations and added into the cell cultures.

Cells exposed to increasing concentrations of PHMB alone or PHMB added individually with nadifloxacin into the wells showed sharp increases in LDH release and a decrease in cells viability (Figure 7a). Using a resazurin assay, we observed that cell viability was affected when exposed to $32 \mathrm{mg} / \mathrm{L}$ of free PHMB, or added individually with nadifloxacin (Figure $7 \mathrm{~b}$ ). In contrast, the exposure to nadifloxacin did not induce LDH release, and the cells' viability was not affected throughout the experiment. These findings confirm a direct relationship between the concentration of free PHMB and the toxic effect on keratinocytes. On the other hand, these results also indicate that PHMB and nadifloxacin added individually into the wells do not assemble into nanoparticles, and therefore remain as free compounds. In contrast, cells exposed to increasing concentrations of PHMB/nadifloxacin nanoparticles showed a slower release of $\mathrm{LDH}$, with cell viability affected at only the highest level tested $(64 / 128 \mathrm{mg} / \mathrm{L}$ ) (Figure $7 \mathrm{~b}$ ). These results suggest that formulating PHMB and nadifloxacin as nanoparticles can reduce PHMB toxicity towards keratinocytes. The toxic effects of nanoparticles at higher concentrations could be due to the free PHMB. Further optimization to increase encapsulation efficiency can lower the amount of free PHMB in the formulation, and hence further decrease its toxic effects towards keratinocytes. 

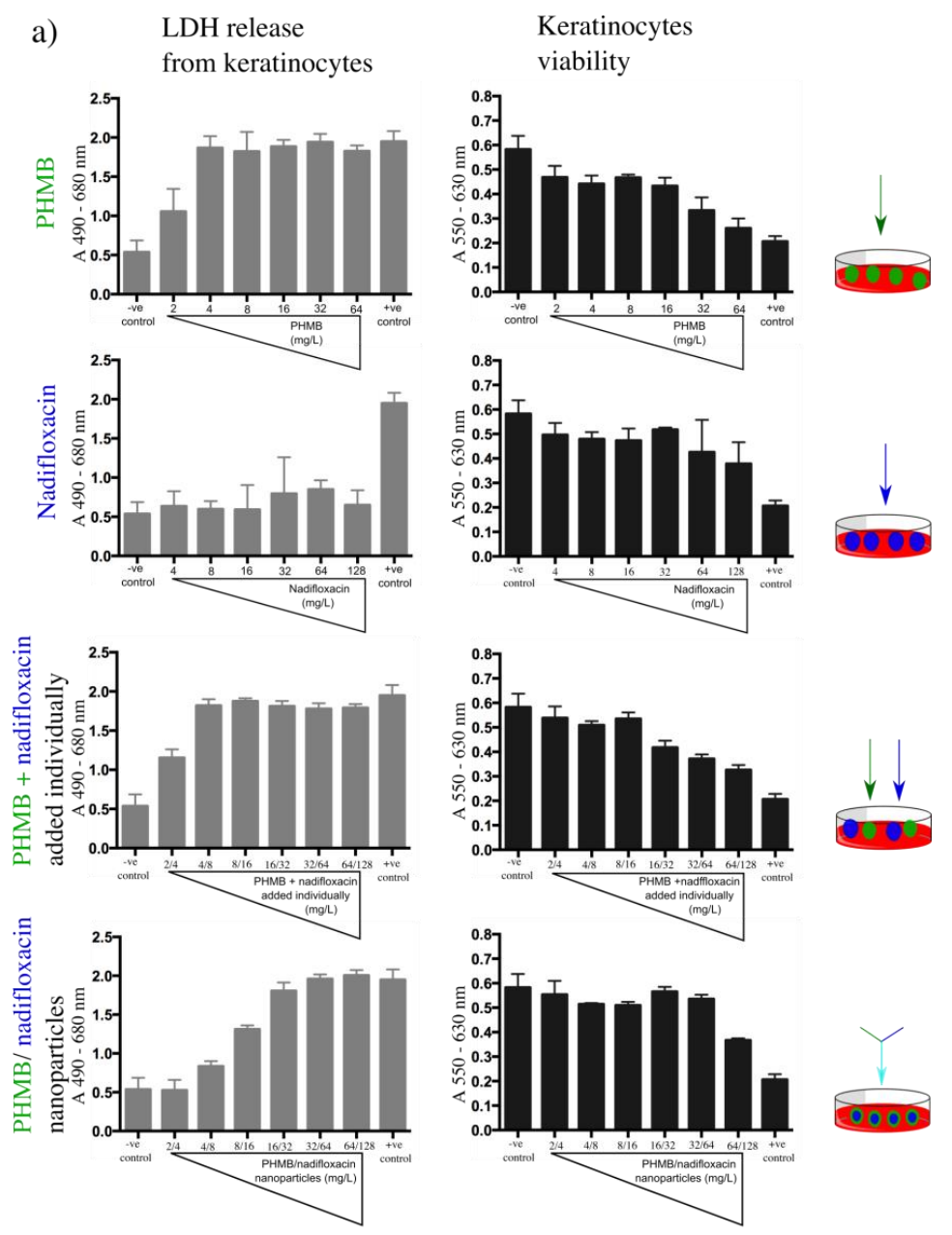

b)

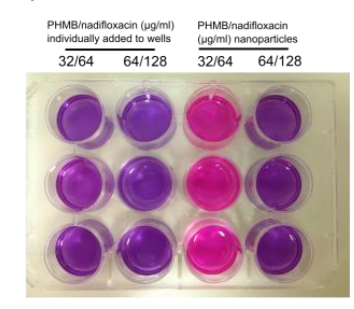

c)

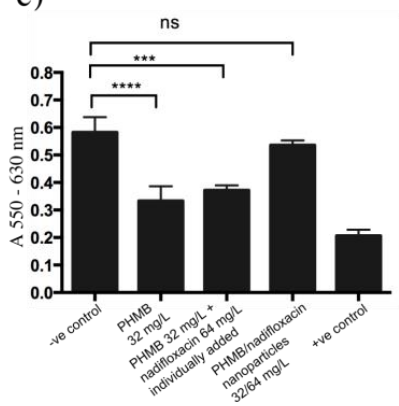

Figure 7. Toxicity effects of nanoparticles towards keratinocytes. Keratinocytes were exposed to increasing concentration of PHMB or nadifloxacin, alone and in combinations. The combinations were either added individually to the wells or formulated as nanoparticles. (a) The toxicity assessment was based on the amount of LDH released by keratinocytes, followed by evaluation of cell viability using resazurin assay. Untreated cells were used as the base for LDH released by the cells and for viability resazurin assay (negative control). Cells treated with $0.5 \%$ Triton- $X 100$ were used as the positive control. (b) Image taken on cells exposed to different antimicrobial formulation and subjected to resazurin assay. The color of resazurin added into the cells treated with $32 \mathrm{mg} / \mathrm{L}$ of PHMB and $64 \mathrm{mg} / \mathrm{L}$ nadifloxacin added individually into the wells (column 1) remained purple indicates cell death. As expected, the same was observed with higher concentrations of PHMB $(64 \mathrm{mg} / \mathrm{mL})$ and nadifloxacin $(128 \mathrm{mg} / \mathrm{mL}$ ) (column 2). The same effects were observed when cells were treated with PHMB alone at $32 \mathrm{mg} / \mathrm{mL}$ (image is not shown). (c) Statistical analysis on the viability of the cells when exposed to $32 \mathrm{mg} / \mathrm{L}$ of PHMB alone, individually added with nadifloxacin or pre-formulated as nanoparticles. No significant difference in cell viability when treated with $\mathrm{PHMB} /$ nadifloxacin nanoparticles at $32 \mathrm{mg} / \mathrm{L}$ compared to the non-treated cells. Error bars represent standard deviations. $* * *(p \leq 0.001),{ }^{* * * *}(p \leq 0.0001)$, ns (not significant). 


\section{Discussion}

To our knowledge, this is the first work that reports PHMB interactions with small molecule antibiotics. We hypothesized that the spontaneous formation of PHMB/nadifloxacin nanoparticles were due to direct interactions between both compounds, or through indirect interactions promoted by the environment of the compounds. These factors may contribute to the spontaneous formation of PHMB/nadifloxacin nanoparticles. PHMB is an amphiphilic polyelectrolytes compound, built of repeated hydrophobic hexamethylene groups separated by a hydrophilic biguanide segment, that can form hairpin or micelles like structures. Such structures could provide encapsulation space in its hydrophobic core for a hydrophobic compound such as nadifloxacin [35]. Since the formulation was prepared at $\mathrm{pH} 12$ (nadifloxacin was dissolved in $0.1 \mathrm{M} \mathrm{NaOH}$ ), there is a possibility for ionic interactions between the positively charged biguanide of the PHMB and the negatively charged carboxylate anion of nadifloxacin. This carboxyl group would be in its basic deprotonated form, $\mathrm{COO}^{-}$ because the $\mathrm{pH}$ is much higher than the $\mathrm{pKa}$ values of carboxylic acids which are around $4-5$. In this case, we believe that the $\mathrm{pKa}$ of the carboxyl group would be lowered due to the stabilizing effect of the conjugated double bonds on the negative charge of the oxygen, and so at $\mathrm{pH} 12$ virtually all nadifloxacin would be in its carboxylate anion form, which could promote interactions. Additionally, hydrophobic interactions could occur between the hexamethylene moiety of PHMB and the hydrophobic ring structure of nadifloxacin. Formulation at $\mathrm{pH} 12$ ensured the neutrality of the tertiary nitrogens between the cycles, as the pKa values of conjugate acids of tertiary amines was lower than 12 . Moreover, the lone pairs of these nitrogens are delocalized in the aromatic ring, rendering protonation of their aliphatic counterparts. Therefore, the basic neutral form of these amine nitrogens prevented any potential electrostatic repulsion of the positively charged biguanide that could destabilize the complex. Finally, there was also the possibility of formation of hydrogen bonding between the lone pairs of the oxygens and fluorine on nadifloxacin with the hydrogens covalently bound to nitrogen in the biguanide groups. Overall, these favorable interactions may explain why we achieved good encapsulation efficiency $(\sim 58 \%)$ of nadifloxacin within nanoparticles. In our previous study, we demonstrated interactions between PHMB and other molecules. For example, PHMB interactions with CpG ODN forming nanoparticles improved CpG ODN delivery into macrophages [29]. The ability of PHMB to establish interactions with drugs and oligonucleotides suggests that this polymer is a flexible platform for combinations drug reformulation.

The self-assembled PHMB/nadifloxacin nanoparticles displayed desirable physical properties that could promote uptake into mammalian cells. A monodisperse population of nanoparticles of size $<500 \mathrm{~nm}$ is considered suitable for endocytic uptake and vesicle entrapment by the mammalian cells [36]. Additionally, the positive charge of the PHMB/nadifloxacin nanoparticles could promote electrostatic interactions with the negative charge of the cell membrane, further enhancing nanoparticles attachment on the cells' surface and help the invagination process [37]. Furthermore, the asymmetrical spherical shape of the nanoparticles could provide large surface area for attachment onto the cells; increasing the rates of uptake into the cells [36] demonstrated that the rates of uptake for asymmetrical nanoparticles into HeLa cells are four times faster compared to the symmetrical nanoparticles.

The potent antimicrobial effects of PHMB:nadifloxacin nanoparticles against intracellular bacteria and their ability to prevent bacterial re-growth could be due to antimicrobial activity of both components and features of the particles. PHMB's antimicrobial activities appear to be due, in part, to the membrane destabilization and cellular leakages due to the interaction of the biguanide groups with cytoplasmic membranes, lipopolysaccharides, and peptidoglycan of the bacterial cell wall [38]. Also, the hexamethylene segment can interact with phospholipids on the membrane, causing a phase separation that disturbs random distribution of lipids, further destabilizing the membrane structure [39]. Furthermore, recent findings in our laboratory demonstrated that PHMB can enter bacteria cells and condense bacterial chromosomes [28]. PHMB has a strong affinity towards DNA which is believed due to the strong electrostatic interaction between the negatively charged phosphate 
backbone of DNA and the cationic charged of polymer PHMB [29]. Additionally, nadifloxacin antibacterial activities is due to the inhibition of DNA gyrase, interrupting the cleavage-religation during the DNA replication process [40,41]. Thus, combination of PHMB and nadifloxacin effectively kill and prevent re-growth of the bacteria.

Combinations of drugs have long been applied in the pharmaceutical industry as a strategy to enhance their therapeutic effect and reduce toxicities. Here we demonstrated that combinations of PHMB and nadifloxacin formulated as nanoparticles were more potent than the free antimicrobials used alone or in combination, (i.e., without pre-formulation as nanoparticles). The nanoparticles effectively killed intracellular MRSA at the lower dose, prevented re-growth of the bacteria during $72 \mathrm{~h}$ of treatment, improved recovery of the infected keratinocytes, and finally, showed less toxicity towards the host cells compared to other formulations tested. While this study is at an early stage, the results indicate that the nanoparticles tested can provide additional benefits that go beyond the benefits of simple co-administration. It is interesting to note that combinations of PHMB and nadifloxacin as nanoparticles showed potent antimicrobial activities towards intracellular MRSA and the same time reduced PHMB toxicity towards the host cells. Currently, it is unknown why the nanoparticles reduced PHMB toxicity toward the mammalian cells. However, based on our understanding of the PHMB structure, we anticipated that toxicity of PHMB toward the mammalian cells could be due to the interaction of the long chain polymer, which is built of hexamethylene and biguanide moiety with cell membranes. Formulation of PHMB with nadifloxacin produced a sphere-like nanoparticles that could reduce membrane interactions and damage. Nevertheless, additive antimicrobial effects of PHMB/nadifloxacin nanoparticles against intracellular MRSA and prevention of the bacterial re-growth suggest that formulations of nanoparticles did not reduce PHMB potent activities against intracellular MRSA. This is in agreement with a previous study that showed a combination of PHMB with phosphatidylcholine maintained its antimicrobial activities against extracellular S. aureus and Pseudomonas aeruginosa with reduced toxicity effects towards fibroblast [42].

\section{Conclusions}

In conclusion, there are four main findings in this study. Firstly, PHMB can be formulated with nadifloxacin forming nanoparticles via a self-assembly method. Secondly, PHMB/nadifloxacin nanoparticles retained potency of PHMB and nadifloxacin when tested against intracellular S. aureus. Thirdly, PHMB/nadifloxacin nanoparticles can improve recovery of infected cells. Finally, formulations of nanoparticles can reduce the toxicity of PHMB, compared to its use either as a free component alone or in combination with nadifloxacin. Therefore, these findings encourage further investigations of $\mathrm{PHMB}$ / antibiotic combinations as nanoparticles formulations with improved efficacy/toxicity profiles.

Author Contributions: N.F.K., M.d.F.P., and A.C. designed and performed the experiments; N.F.K., M.d.F.P., A.C., and L.G. wrote the manuscript.

Funding: This research and N.F.K. were funded by SLAB Scheme, Ministry of Education, Malaysia, 851209035040. M.d.F.P. was funded by Maplethorpe Postdoctoral Fellowships of the University of London. A.C. was funded by BBSRC LIDo studentship 156780.

Acknowledgments: We thank Mark Turmaine for his assistance with electron microscopy usage, Andrew Hibbert for his assistance with light microscope usage, and Klaudia Kloc Muniak for her assistance in cell culture maintenance. The microscopy work was conducted in Biosciences EM Facility, University College, London and Imaging Suite Facility, Royal Veterinary College, London.

Conflicts of Interest: The authors declare no conflict of interest. The funding sponsors had no role in the design of the study; in the collection, analyses, or interpretation of data; in the writing of the manuscript, and in the decision to publish the results.

\section{References}

1. Esposito, S.; Noviello, S.; Leone, S. Epidemiology and microbiology of skin and soft tissue infections. Curr. Opin. Infect. Dis. 2016, 29, 109-115. [CrossRef] [PubMed] 
2. Karanika, S.; Kinamon, T.; Grigoras, C.; Mylonakis, E. Colonization with Methicillin-resistant Staphylococcus aureus and Risk for Infection Among Asymptomatic Athletes: A Systematic Review and Metaanalysis. Clin. Infect. Dis. 2016, 63, 195-204. [CrossRef] [PubMed]

3. Walsh, T.L.; Chan, L.; Konopka, C.I.; Burkitt, M.J.; Moffa, M.A.; Bremmer, D.N. Appropriateness of antibiotic management of uncomplicated skin and soft tissue infections in hospitalized adult patients. BMC Infect. Dis. 2016, 16, 721. [CrossRef] [PubMed]

4. Lee, G.C.; Hall, R.G.; Boyd, N.K.; Dallas, S.D.; Du, L.C. A prospective observational cohort study in primary care practices to identify factors associated with treatment failure in Staphylococcus aureus skin and soft tissue infections. Ann. Clin. Microbiol. Antimicrob. 2016, 15, 58. [CrossRef] [PubMed]

5. Harch, S.A.J.; MacMorran, E.; Tong, S.Y.C.; Holt, D.C.; Wilson, J.; Athan, E.; Hewagama, S. High burden of complicated skin and soft tissue infections in the Indigenous population of Central Australia due to dominant Panton Valentine leucocidin clones ST93-MRSA and CC121-MSSA. BMC Infect. Dis. 2017, 17, 405. [CrossRef] [PubMed]

6. Moghnieh, R.; Alothman, A.F.; Althaqafi, A.O.; Matar, M.J.; Alenazi, T.H. Epidemiology and outcome of invasive fungal infections and methicillin-resistant Staphylococcus aureus (MRSA) pneumonia and complicated skin and soft tissue infections (cSSTI) in Lebanon and Saudi Arabia. J. Infect. Public Health 2017, 10, 849-854. [CrossRef] [PubMed]

7. Macmorran, E.; Harch, S.; Athan, E.; Lane, S.; Tong, S.; Crawford, L. The rise of methicillin resistant Staphylococcus aureus: Now the dominant cause of skin and soft tissue infection in Central Australia. Epidemiol. Infect. 2017, 145, 2817-2826. [CrossRef] [PubMed]

8. Gould, I.M. Antibiotics, skin and soft tissue infection and meticillin-resistant Staphylococcus aureus: Cause and effect. Int. J. Antimicrob. Agents 2009, 34, S8-S11. [CrossRef]

9. Bal, A.M.; David, M.Z.; Garau, J.; Gottlieb, T.; Mazzei, T. Future trends in the treatment of methicillin-resistant Staphylococcus aureus (MRSA) infection: An in-depth review of newer antibiotics active against an enduring pathogen. J. Glob. Antimicrob. Resist. 2017, 10, 295-303. [CrossRef] [PubMed]

10. Grant, S.S.; Hung, D.T. Persistent bacterial infections, antibiotic tolerance, and the oxidative stress response. Virulence 2013, 4, 1-11. [CrossRef] [PubMed]

11. Carryn, S.; Van Bambeke, F.; Mingeot-Leclercq, M.P.; Tulkens, P.M. Activity of beta-lactams (ampicillin, meropenem), gentamicin, azithromycin and moxifloxacin against intracellular Listeria monocytogenes in a $24 \mathrm{~h}$ THP-1 human macrophage model. J. Antimicrob. Chemother. 2003, 51, 1051-1052. [CrossRef] [PubMed]

12. Bambeke, F.V.; Michot, J.; Van Eldere, J.; Tulkens, P.M. Quinolones in 2005: An update. Clin. Microbiol. Infect. 2005, 11, 256-280. [CrossRef] [PubMed]

13. Barcia-Macay, M.; Seral, C.; Mingeot-Leclercq, M.P.; Tulkens, P.M.; Van Bambeke, F. Pharmacodynamic evaluation of the intracellular activities of antibiotics against Staphylococcus aureus in a model of THP-1 macrophages. Antimicrob. Agents Chemother. 2006, 50, 841-851. [CrossRef] [PubMed]

14. Garzoni, C.; Kelley, W.L. Staphylococcus aureus: New evidence for intracellular persistence supplement. Trends Microbiol. 2009, 17, 59-65. [CrossRef] [PubMed]

15. Vesga, O.; Groeschel, M.C.; Otten, M.F.; Brar, D.W.; Vann, J.M.; Proctor, R. a Staphylococcus aureus small colony variants are induced by the endothelial cell intracellular milieu. J. Infect. Dis. 1996, 173, 739-742. [CrossRef] [PubMed]

16. Nguyen, H.A.; Denis, O.; Vergison, A.; Theunis, A.; Tulkens, P.M.; Struelens, M.J.; Bambeke, F.V. tracellular activity of antibiotics in a model of human THP-1 macrophages infected by a Staphylococcus aureus small-colony variant strain isolated from a cystic fibrosis patient: Pharmacodynamic evaluation and comparison with isogenic normal-phenotype a. Antimicrob. Agents Chemother. 2009, 53, 1434-1442. [CrossRef] [PubMed]

17. Wu, L.; Zhang, J.; Watanabe, W. Physical and chemical stability of drug nanoparticles. Adv. Drug Deliv. Rev. 2011, 63, 456-469. [CrossRef] [PubMed]

18. Zhang, L.; Pornpattananangku, D.; Hu, C.M.J.; Huang, C.M. Development of nanoparticles for antimicrobial drug delivery. Curr. Med. Chem. 2010, 17, 585-594. [CrossRef] [PubMed]

19. Miramoth, N.S.; Meo, C.D.; Zouhiri, F.; Saïd-Hassane, F.; Valetti, S.; Gorges, R. Self-assembled squalenoylated penicillin bioconjugates: An original approach for the treatment of intracellular infections. ACS Nano 2012, 6 , 3820-3831. [CrossRef] [PubMed] 
20. Clemens, D.L.; Lee, B.Y.; Xue, M.; Thomas, C.R.; Meng, H.; Ferris, D. Targeted intracellular delivery of antituberculosis drugs to Mycobacterium tuberculosis-infected macrophages via functionalized mesoporous silica nanoparticles. Antimicrob. Agents Chemother. 2012, 56, 2535-2545. [CrossRef] [PubMed]

21. Zou, A.; Li, Y.; Chen, Y.; Angelova, A.; Garamus, V.M.; Li, N.; Drechsler, M.; Angelov, B.; Gong, Y. Self-assembled stable sponge-type nanocarries for Brucea javanica oil delivery. Coll. Surf. B 2017, 153, 310-319. [CrossRef] [PubMed]

22. Ventosa, N.; Ferrer-Tasies, L.; Moreno-Calvo, E. Quatsomes: Highly stable nanovesicles formed by sterols and. Tech. Proc. 2013, 1, 634-637.

23. Cano-Sarabia, M.; Angelova, A.; Ventosa, N.; Lesieur, S.; Veciana, J. Cholesterol induced CTAB micelle-to-vesicle phase transitions. J. Coll. Inter. Sci. 2010, 350, 10-15. [CrossRef] [PubMed]

24. Angelova, A.; Ionov, R.; Koch, M.H.J.; Rapp, G. Interaction of the peptide antibiotic alamethicin with bilayer-and non- bilayer-forming lipids: Influence of increasing alamethicin concentration on the lipids supramolecular structures. Arch. Biochem. Biophys. 2000, 378, 93-106. [CrossRef] [PubMed]

25. Angelov, B.; Angelova, A.; Filippov, S.K.; Drechsler, M.; Štěpánek, P.; Lesieur, S. Multicompartment lipid cubic nanoparticles with high protein upload: Millisecond dynamics of formation. ACS Nano 2014, 8, 5216-5226. [CrossRef] [PubMed]

26. Zerkoune, L.; Lesieur, S.; Putaux, J.L. Mesoporous self-assembled nanoparticles of biotransesterified cyclodextrins and nonlamellar lipids as carriers of water-insoluble substances. Soft Matter 2016, 12, 7539-7550. [CrossRef] [PubMed]

27. Guerzoni, L.P.B.; Nicolas, V.; Angelova, A. In Vitro Modulation of TrkB Receptor Signaling upon Sequential Delivery of Curcumin-DHA Loaded Carriers Towards Promoting Neuronal Survival. Pharm. Res. 2017, 34, 492-505. [CrossRef] [PubMed]

28. Chindera, K.; Mahato, M.; Kumar Sharma, A.; Horsley, H. The antimicrobial polymer PHMB enters cells and selectively condenses bacterial chromosomes. Sci. Rep. 2016, 6, 23121. [CrossRef] [PubMed]

29. Firdessa, R.; Good, L.; Amstalden, M.C.; Chindera, K. Pathogen- and Host-Directed Antileishmanial Effects Mediated by Polyhexanide (PHMB). PLoS Negl. Trop. Dis. 2015, 9, e0004041. [CrossRef] [PubMed]

30. Gala, R.P.; D'Souza, M.; Zughaier, S.M. Evaluation of various adjuvant nanoparticulate formulations for meningococcal capsular polysaccharide-based vaccine. Vaccine 2016, 34, 3260-3267. [CrossRef] [PubMed]

31. Kamaruzzaman, N.F.; Firdessa, R.; Good, L. Bactericidal effects of polyhexamethylene biguanide against intracellualar Staphylococcus aureus EMRSA-15 and USA 300. J. Antimicrob. Chemother. 2016, 71, 1252-1259. [CrossRef] [PubMed]

32. Katas, H.; Alpar, H.O. Development and characterisation of chitosan nanoparticles for siRNA delivery. J. Control. Release 2006, 115, 216-225. [CrossRef] [PubMed]

33. Kamaruzzaman, N.F.; Chong, S.Q.Y.; Edmondson-Brown, K.M.; Ntow-Boahene, W.; Bardiau, M.; Good, L. Bactericidal and anti-biofilm effects of polyhexamethylene Biguanide in models of intracellular and biofilm of Staphylococcus aureus isolated from bovine mastitis. Front. Microbiol. 2017, 8, 1-10. [CrossRef] [PubMed]

34. Soong, G.; Chun, J.; Parker, D.; Prince, A. Staphylococcus aureus activation of caspase 1/calpain signaling mediates invasion through human keratinocytes. J. Infect. Dis. 2012, 205, 1571-1579. [CrossRef] [PubMed]

35. De Paula, G.F.; Netto, G.I.; Mattoso, L.H.C. Physical and chemical characterization of poly(hexamethylene biguanide) hydrochloride. Polymers 2011, 3, 928-941. [CrossRef]

36. Gratton, S.; Ropp, P.; Pohlhaus, P.; Luft, C.; Madden, V.; Napier, M.; DeSimone, J. The effect of particle design on cellular internalization pathways. ACS Nano 2015, 201, 3458-3461. [CrossRef] [PubMed]

37. Kou, L.; Sun, J.; Zhai, Y.; He, Z. The endocytosis and intracellular fate of nanomedicines: Implication for rational design. Asian J. Pharm. Sci. 2013, 8, 1-10. [CrossRef]

38. Gilbert, P.; Moore, L.E. Cationic antiseptics: Diversity of action under a common epithet. J. Appl. Microbiol. 2005, 99, 703-715. [CrossRef] [PubMed]

39. Broxton, P.; Woodcock, P.; Heatley, F.; Gilbert, P. Interaction of some polyhexamthylene diguanides and membrane phospholipids in Escherichia coli. J. Appl. Bacteriol. 1984, 57, 115-124. [CrossRef] [PubMed]

40. Collin, F.; Karkare, S.; Maxwell, A. Exploiting bacterial DNA gyrase as a drug target: Current state and perspectives. Appl. Microbiol. Biotechnol. 2011, 92, 479-497. [CrossRef] [PubMed] 
41. Oizumi, N.; Kawabata, S.; Hirao, M.; Watanabe, K.; Okuno, S.; Fujiwara, T.; Kikuchi, M. Relationship between mutations in the DNA gyrase and topoisomerase IV genes and nadifloxacin resistance in clinically isolated quinolone-resistant Staphylococcus aureus. J. Infect. Chemother. 2001, 7, 191-194. [CrossRef] [PubMed]

42. Müller, G.; Kramer, A.; Schmitt, J.; Harden, D.; Koburger, T. Reduced cytotoxicity of polyhexamethylene biguanide hydrochloride (PHMB) by egg phosphatidylcholine while maintaining antimicrobial efficacy. Chem. Biol. Interact. 2011, 190, 171-178. [CrossRef] [PubMed] 